\title{
EVALUATION OF FETAL REACTIVITY IN A TEST WITH ACOUSTIC STIMULATION IN WOMEN WITH A HISTORY OF CESAREAN SECTION
}

\author{
Larysa Nazarenko ${ }^{1}$, Lyudmyla Huk ${ }^{2}$, Nataliia Nestertsova ${ }^{3}$ \\ ${ }^{1}$ Department of Genetic, Obstetrics, Gynecology and Fetal Medicine, Kharkiv Medical Academy of Postgraduate Education, Kharkiv, \\ Ukraine \\ dr.lgn@ukr.net \\ ORCID: http://orcid.org/0000-0001-6064-8026 \\ ${ }^{2}$ Department of Genetic, Obstetrics, Gynecology and Fetal Medicine, Kharkiv Medical Academy of Postgraduate Education, Kharkiv, \\ Ukraine \\ gyklyudmila@gmail.com \\ ORCID: http://orcid.org/0000-0001-9573-3829 \\ ${ }^{3}$ Department of Genetic, Obstetrics, Gynecology and Fetal Medicine, Kharkiv Medical Academy of Postgraduate Education, Kharkiv, \\ Ukraine \\ n.nestertsova@gmail.com \\ ORCID: http://orcid.org/0000-0003-3098-9641
}

ARTICLE INFO

ABSTRACT

Article history:

Received date 22.09 .2020

Accepted date 26.10.2020

Published date 30.10 .2020

Object of study: pregnancy in women with a history of cesarean section, perinatal period. The problem to be solved: clarification of the perinatal significance of the factor Section:

Practical medicine "scarred uterus", substantiation of the clinical significance of the test with acoustic stimulation of the fetus as a method for predicting early neonatal adaptation.

Main scientific results: it was found that pregnancy in women with a history of ce-

D O I sarean section is characterized by an increased perinatal risk and is associated with

$10.21303 / 2313-8416.2020 .001482$

KEYWORDS psychoemotional stress of the mother, which affects the reactivity of fetal cerebral hemodynamics. It has been shown that the test with acoustic stimulation of fetus allows one to

cesarean section

scarred uterus

fetal condition

antenatal assessment

perinatal pathology gain an idea of the fetus's perception of stressful influences, which should be taken into account when drawing up a delivery plan.

The area of practical use of research results: obstetric departments of maternity hospitals and multidisciplinary hospitals.

An innovative technological product: technology for assessing the state of the fetus in full-term pregnancy, which allows predicting complications of early neonatal adaptation based on the susceptibility of the fetus in the test with acoustic stimulation.

Scope of application of an innovative technological product: obstetric clinical practice, perinatal medicine.

(C) The Author(s) 2020. This is an open access article under the CC BY license http://creativecommons.org/licenses/by/4.0).

\section{Introduction}

\subsection{Object of study}

Pregnancy in women with a history of cesarean section, perinatal period.

\subsection{Description of the problem}

Delivery by caesarean section (CS) is recognized worldwide as the most effective way to overcome the risks of perinatal losses, as a guarantee of reducing maternal morbidity and mortality [1]. However, this does not hide the obvious problems associated with the increase in CS levels throughout the world [2].

In the modern reproductive population, the number of women with uterine scar after CS has increased significantly. Many of them are planning a new pregnancy in the future. The postponed surgical intervention leaves negative consequences: pain and discomfort in the area of the scar, inflammatory changes in the pelvic organs, disorders of the immune and endocrine systems, sexual dysfunction [3]. These factors serve as prerequisites for the development of a number of complications during subsequent pregnancy.

Traditionally, the greatest importance is attached to the reliability of the scar during labor. At the same time, an equally important aspect is perinatal, which is not given enough attention in clinical practice. The scarred uterus, which has experienced trauma and reparative process, theo- 
retically is no longer an ideal host, which inevitably affects the conditions for development of the fetus. This aspect remains poorly understood.

\subsection{Suggested way to solve the problem}

Psychophysiological disorders are a particular problem in women with a CS in anamnesis [4]. Patients with negative experience of previous childbirth have a higher risk of stress, a complex of neuropsychiatric disorders [5].

Maternal stress, regardless of its causes, is a risk factor for perinatal pathology [6], associated with physiological [7], neurological, psychological consequences in offspring [8,9].

All of the above substantiates the relevance of assessing the adaptive potential of the fetus when planning the method of delivery - choosing a repeated CS or vaginal delivery. The solution to this problem is possible under the conditions of carrying out functional tests [10, 11]. One of the promising options for testing resistance to stress is the test with acoustic stimulation (TAS) of the fetus $[12,13]$. Sound is a stressful stimulus, the target of which is brain $[14,15]$. Acoustic stimulation is one of the factors of direct action on the fetus, with the ability to directly assess its sensory response $[16,17]$.

The aim of this work is to assess the prognostic value of the fetal acoustic stimulation test by assessing changes in fetal cerebral blood flow during full-term pregnancy in women with CS in anamnesis, in comparison with indicators characterizing the psycho-emotional status of a woman.

\section{Materials and research methods}

The studies were carried out in 128 multiparous women with singleton pregnancies in the period of 37-39 weeks during 2017-2019 on the basis of the municipal non-profit enterprise "City Clinical Maternity Hospital No. 6" of the Kharkiv City Council. According to the criterion "presence of scarred uterus", two clinical groups were formed, selected according to the "case-control" ("copy-pair") principle. The first group included 64 pregnant women with one history of CS, with an intergenetic interval of 2-7 years. The indications for CS in the past were obstructive labor (in 28), fetal compromise (in 21), premature placental abruption (6), breech presentation (5), severe preeclampsia (4). The only significant perinatal risk factor was the uterine scar. The second (control) group consisted of 64 multiparous women with an intact uterus, with an intergenetic interval of $2-15$ years.

Exclusion criteria were fetal malformations, abnormalities and tumors of the uterus, somatic diseases, and neurological disorders.

The age of women in group 1 was on average $32.1 \pm 3.8$ years (from 26 to 39), in group 2 $30.2 \pm 5.1$ years (from 25 to 40 ).

The state of the feto-placental complex was assessed according to the data of fetometry, placentography, dopplerometry of the uteroplacental-fetal vascular circuit, with measurement of blood flow in the middle cerebral artery (MCA), with the determination of vascular resistance indicators.

To achieve the goal, a test with acoustic stimulation was chosen, known as "prenatal hearing screening" M. Sovilj, A. Ljubic [12]. The studies were carried out on a Mindray DC-T6 apparatus (China) with a convex transabdominal sensor with a radiation frequency of $3.5 \mathrm{MHz}$, with a color marking of blood flows, during periods of absence of respiratory and motor activity in the fetus. Qualitative analysis of Doppler images included the definition of vascular resistance indicators: systolic-diastolic ratio (SDR), pulsation index (PI), resistance index (RI).

The test procedure with acoustic stimulation (TAS) included, in particular, the use of antiphones on the ears of a pregnant woman in order to avoid the influence of sound stimulation through the mother's auditory system, recording the baseline Doppler curve MCA, the delivery of sound stimulus (intensity $90 \mathrm{~dB}$, frequency $1500-4500 \mathrm{~Hz}$, duration $0.2 \mathrm{sec}$.) on the area of the fetal auricle at a right angle at a distance of $5 \mathrm{~cm}$ from the abdomen of the pregnant woman, recording the Doppler curve MCA.

To assess the response of fetal cerebral blood flow, we used the coefficient of vascular reactivity (CVR) - the ratio of the initial SDR MCA to the SDR of the same vessel after the acoustic signal is applied: $\mathrm{CVR}=\mathrm{SDR}_{\text {initial }} / \mathrm{SDR}_{\text {after the acoustic signal }}$. When interpreting the changes occurring in each observation, it was taken into account that CVR $>1$ reflects a decrease in the vascular resis- 
tance of the MCA, respectively, a dilatation of the vessel. With CVR $<1$ - an increase in vascular resistance, vasospasm.

Psycho-diagnostic examination was carried out in the following volume: multifactorial personality questionnaire FPI "Freiburg Personality Inventory", test questionnaire for measuring levels of anxiety by J. Taylor, adapted by T. A. Nemchinov, self-assessment of the level of reactive and personal anxiety by Ch. D. Spielberger and Yu. L. Khanin, express diagnostics of anxiety in the Luscher color choices test.

The primary endpoint was development of fetal compromise during labor, birth of a baby with a low Apgar score, need for neonatal care and intensive care.

The study design was reviewed by the Ethics Commission of the "City Clinical Maternity Hospital No. 6" KCC, approved by the Ethics Committee of the KhMAPE, recognized as consistent with the principles of the Declaration of Helsinki of the General Assembly of the World Medical Association (1964-2000), the Council of Europe Convention on Human Rights and Biomedicine (1997), the international code of medical ethics and the rights of Ukraine. All patients provided informed consent for additional examinations on a conscientious basis.

The results were statistically processed using the STATISTICA-7 software package. Qualitative data were presented as a percentage; quantitative data were presented as mean and standard error $(M \pm m)$. To compare quantitative indicators in independent samples, the Student's t-test was used. The critical significance level for testing statistical hypotheses in the study was taken equal to 0.05 .

\section{Results}

In all cases in both groups, the Doppler images had a typical biphasic form, consisting of an acceleration phase (ascending part), reflecting an increase in blood flow velocity corresponding to the heart systole, and a deceleration phase (descending branch), reflecting a diastolic decrease in blood flow.

The average baseline indicators of fetal cerebral blood flow resistance (Table 1) did not reveal differences in the groups. As you can see, all three indices are identical to each other. At the same time, it can be noted that for real clinical practice, it is the SDR that is most acceptable for quick, simple calculations, since it has a slightly larger range due to integers. At the same time, PI and RI, in contrast to SDR, have values that differ in the decimal order. This justified our choice of SDR to assess the dynamics of cerebral blood flow in the TAS and calculate the CVR.

The study of the psycho-emotional sphere of pregnant women was carried out before performing the TAS.

A general survey of women in group 1 showed that $48.4 \%$ had a distinct negative psychological attitude towards vaginal delivery and a belief in the inevitability of repeated CS. In group 2, $4.7 \%$ of women had no alternative attitude towards CS due to negative experience of the first birth. In group 1, 35.9\% of women were hospitalized during pregnancy due to the threat of miscarriage or for prophylactic purposes.

Table 1

Indicators of vascular resistance in the middle cerebral artery of the fetus in the groups of multiparous women with scarred (1) and intact uterus (2)

\begin{tabular}{cccc}
\hline Group & Systole-diastolic ratio & Pulse index & Resistance index \\
\hline 1 & $4.99 \pm 0.133$ & $1.52 \pm 0.05$ & $0.78 \pm 0.05$ \\
2 & $4.63 \pm 0.123$ & $1.5 \pm 0.04$ & $0.82 \pm 0.05$
\end{tabular}

Evaluating the response of fetal cerebral blood flow to an acoustic stimulus, we identified several options. First of all, depending on the presence/absence of SDR changes by more than $5 \%$ from the baseline CVR value, the results were divided into reactive and non-reactive TAS categories.

The areactive test - the absence of changes in the SDR indices in the MCA after an acoustic stimulus by more than $5 \%$ from the initial one - in group 1 we stated in 19 cases (29.7\%), in group 2 in $10(15.6 \%)$. In both groups, areactive fetal TAS is associated with negative individual characteristics of women. They were distinguished by a high level of neuroticism, emotional lability, susceptibility, with mood swings, expressive emotional reactions, as well as weakened self-control and higher personal anxiety. 
In group 2, 8 out of 10 women with areactive fetal TAS gave birth to children with an Apgar score of 5-7 points due to various clinical situations during childbirth $(12.5 \%$ of the number of women in the group). In group 1, all women with areactive TAS underwent CS, including in 15 cases elective planned repeated at the request of the woman, in 4 - emergency due to the development of fetal compromise in the latent phase of labor.

The condition of children born to women of group 1, in whom the fetus showed areactive TAS, was satisfactory at birth, 8-9 points on the Apgar scale. However, in the further course of the neonatal period, 9 cases developed CNS excitation syndrome, a disorder of early neonatal adaptation.

The reactive test consisted of obtaining a response in the form of a change in the SDR in the MCA. In this case, an increase in vascular resistance, reflecting vasospasm, which causes a decrease in the intensity of blood flow in relation to the initial (baseline) level, we consider as a negative reaction. At the same time, we interpreted a decrease in vascular resistance, which means dilatation of the vessel and an increase in blood flow, as a positive reaction.

In our observations, the systolic spectrum underwent changes in most cases, while the diastolic spectrum remained practically unchanged. Absent or reverse diastolic blood flow was not recorded in any case.

When repeating dopplerometry in the interval of 10-15 minutes after acoustic stimulation, we noted the reversible nature of the changes.

We observed reactive TAS in 99 cases (77.3\% of the total number of women). Comparative analysis of changes in blood flow resistance indicators in MCA in groups of multiparous women is presented in Table 2.

\section{Table 2}

Comparative analysis of vascular reactivity indicators of the middle cerebral artery of the fetus in the test with acoustic stimulation

\begin{tabular}{|c|c|c|c|c|}
\hline \multirow{2}{*}{ Group } & \multirow{2}{*}{$N$} & \multicolumn{2}{|c|}{ Systole-diastolic ratio (SDR) } & \multirow{2}{*}{ CVR } \\
\hline & & Baseline indicator & Responce in TAS & \\
\hline 1 & 64 & $4.99 \pm \cdot .133$ & $5.3 \pm 0.121$ & $0.87 \pm 0.022 *$ \\
\hline 2 & 64 & $4.63 \pm \cdot .123$ & $4.7 \pm 0.11$ & $1.04 \pm 0.021$ \\
\hline
\end{tabular}

Note: ${ }^{*}$ - significance of differences at $p<0.001$ when comparing groups $1-2$

The data given in Table 2 demonstrate that for group 1 as a whole, changes in fetal cerebral hemodynamics are characteristic, corresponding to vasospasm. The absence of differences in the response to the acoustic stimulus in group 2 is a manifestation of the presence of opposite changes, which was reflected in the mean values. A slightly higher SDR in the response in group 1, despite the lack of significant differences, corresponds to the fact that in a greater number of observations there was spasm (ISR $>1)$.

An individual analysis of clinical observations allowed us to note that the TAS response towards a maximum increase in SDR, respectively, a decrease in CVR up to $20 \%$ coincides with impairments in early neonatal adaptation in newborn children. They had a syndrome of excitement or, on the contrary, depression of the central nervous system, retention of fetal fluid in the alveoli, pathological weight loss and delayed weight recovery, and transient tachypnea was observed. In four such cases, there were meconium-stained amniotic fluid ( 2 in each group) without complications from the fetus, in 2 cases meconium-stained amniotic fluid resulted in aspiration.

\section{Discussion}

The fact that the mean baseline values of fetal cerebral blood flow resistance did not reveal differences in the groups confirms the existing opinion about the low value of the isolated determination of blood flow in MCA [18].

The higher hospitalization rate during pregnancy in group 1 patients appears to be a factor with a negative impact on the fetus. Probably, against the background of treatment of a woman in a hospital, psycho-emotional stress developed or worsened. This fact can be considered a kind of 
stress-generating factor contributing to the formation of somatoform disorders against the background of a "special disease" - a scar on the uterus $[4,5]$.

The observed dynamics of MCA reactivity under the influence of an acoustic stimulus reflects the gradient of changes in the velocity of blood movement in systole and diastole. The more the difference between the systolic and diastolic, as well as the average, increases, the more the resistance indices increase. The presence of a reaction in the TAS reflects the susceptibility of the fetus to stress.

In our observations, the systolic spectrum underwent changes in most cases, while the diastolic spectrum remained practically unchanged. Absent or reverse diastolic blood flow was not recorded in any case. The reversible nature of the changes and the absence of signs of critical shifts in fetal cerebral hemdynamics suggests that the supplied acoustic signal does not belong to the number of traumatic factors, does not pose a danger and does not affect the development of the child, but is a signal that the fetus "copes" with, demonstrating its ability perception of stress. The lack of response can be explained by the fact that the study was carried out in the sleep phase, which is not always possible to take into account based on the subjective feelings of the woman. It is also possible the influence of some technical aspects of the test, as well as the woman's characteristics, for example, excess subcutaneous tissue, the amount of amniotic fluid, since the amniotic environment and adipose tissue play the role of a filter in the perception of external sound stimuli [19].

The average data obtained in the presented study demonstrated a general pattern, which consists in an increase in the vascular resistance of the cerebral vessels of the fetus in the group of women with an scarred uterus, in contrast to multiparous women with an intact uterus. In general terms, this fact reflects a trend towards increased perinatal risk in women with a history of CS. The ability to predict disorders of neonatal adaptation with the help of TAS, in the development of which the state of the nervous system of newborns is important, contributes to the decision of the choice of the optimal model of delivery.

In addition to the perinatal significance of the functional test under consideration, it is also necessary to take into account that the areactive TAS provides a basis for including a particular intrauterine patient in the risk group for speech and hearing impairment [20].

Study limitations. The present study was carried out in a homogeneous limited clinical group and does not cover other types of obstetric and perinatal pathology and women with other perinatal risk factors (breech presentation of the fetus, overweight women, with anemia, preeclampsia), which at this stage does not allow us to judge the specific or the universal nature of the identified changes.

Prospects for further research. Investigation of changes in fetal cerebral hemodynamics in the test with acoustic stimulation of the fetus in other clinical groups (for example, in breech presentation of the fetus, in overweight women, with anemia, preeclampsia). To study the influence of perinatal communications in the mother-fetus dyad as a possible way to optimize the course of the neonatal adaptation period in groups, based on the use of a test with acoustic stimulation of the fetus.

\section{Conclusions}

1. A non-invasive method for assessing the reactivity of the fetus to external sound effects - a test with acoustic stimulation with an assessment of changes in fetal cerebral hemodynamics - allows you to clarify the stability of the fetus under stress conditions that are inevitable during delivery.

2. The response in the form of spasm of the middle cerebral artery or unresponsiveness allows predicting impairments in early neonatal adaptation, which should be taken into account in the management of childbirth, giving priority to the safety of the fetus.

3. Pregnancy in women with an scarred uterus should be considered from the standpoint of increased perinatal risk associated with psycho-emotional stress of the mother, which affects the reactivity of fetal cerebral hemodynamics.

\section{Conflict of interests}

The authors declare that they have no conflicts of interest.

\section{References}

[1] WHO recommendations on non-clinical interventions to reduce unnecessary caesarean sections (2018). World Health Organization, 79. Available at: https:/www.who.int/reproductivehealth/publications/non-clinical-interventions-to-reduce-cs/en/

[2] Dubrova, L. Y. (2013). The improvement of medical support and delivery of women with a previous cesarean section. Kyiv. 
[3] Vdovichenko, Y. P., Gurzhenko, O. Y. (2015). Algorithm of diagnostics, prophylaxis and treatment of sexual disfunctions in women after caesarian section. Zdorovye zhenshiny, 5 (101), 66-68.

[4] Orovou, E., Dagla, M., Iatrakis, G., Lykeridou, A., Tzavara, C., Antoniou, E. (2020). Correlation between Kind of Cesarean Section and Posttraumatic Stress Disorder in Greek Women. International Journal of Environmental Research and Public Health, 17 (5), 1592. doi: http://doi.org/10.3390/ijerph17051592

[5] Garthus-Niegel, S., von Soest, T., Knoph, C., Simonsen, T. B., Torgersen, L., Eberhard-Gran, M. (2014). The influence of women's preferences and actual mode of delivery on post-traumatic stress symptoms following childbirth: a population-based, longitudinal study. BMC Pregnancy and Childbirth, 14 (1). doi: http://doi.org/10.1186/1471-2393-14-191

[6] Garcia-Flores, V., Romero, R., Furcron, A.-E., Levenson, D., Galaz, J., Zou, C. et. al. (2020). Prenatal Maternal Stress Causes Preterm Birth and Affects Neonatal Adaptive Immunity in Mice. Frontiers in Immunology, 11. doi: http://doi.org/10.3389/ fimmu.2020.00254

[7] McCreary, J. K., Truica, L. S., Friesen, B., Yao, Y., Olson, D. M., Kovalchuk, I. et. al. (2016). Altered brain morphology and functional connectivity reflect a vulnerable affective state after cumulative multigenerational stress in rats. Neuroscience, 330 , 79-89. doi: http://doi.org/10.1016/j.neuroscience.2016.05.046

[8] Zakharova, L. A. (2015). Perinatal stress in brain programming and in the pathogenesis of neuropsychiatric diseases. Bulletin of the Russian Academy of Sciences. Biological series, 1, 17-26. doi: http://doi.org/10.7868/s0002332915010130

[9] Entringer, S., Buss, C., Wadhwa, P. D. (2015). Prenatal stress, development, health and disease risk: A psychobiological perspective - 2015 Curt Richter Award Paper. Psychoneuroendocrinology, 62, 366-375. doi: http://doi.org/10.1016/j.psyneuen.2015.08.019

[10] Tan, K. H., Smyth, R. M., Wei, X. (2013). Fetal vibroacoustic stimulation for facilitation of tests of fetal wellbeing. Cochrane Database of Systematic Reviews. doi: http://doi.org/10.1002/14651858.cd002963.pub2

[11] Umana, O. D, Siccardi, M. A. (2020). Prenatal Non-stress Test. Available at: https://www.ncbi.nlm.nih.gov/books/NBK537123/

[12] Sovili, M., Ljubic, A. (1992). Possibilities of Prenatal Examination of Reaction to Sound in fetuses with Congenital Infections. Journal of Perinatal Medicine section, Serbian Medical Society, 17-18.

[13] Jardri, R., Houfflin-Debarge, V., Delion, P., Pruvo, J., Thomas, P., Pins, D. (2011). Assessing fetal response to maternal speech using a noninvasive functional brain imaging technique. International Journal of Developmental Neuroscience, 30 (2), $159-161$. doi: http://doi.org/10.1016/j.ijdevneu.2011.11.002

[14] Selander, J., Rylander, L., Albin, M., Rosenhall, U., Lewné, M., Gustavsson, P. (2019). Full-time exposure to occupational noise during pregnancy was associated with reduced birth weight in a nationwide cohort study of Swedish women. Science of The Total Environment, 651, 1137-1143. doi: http://doi.org/10.1016/j.scitotenv.2018.09.212

[15] Dumov, E. L., Nöcker-Ribaupierre, M., Andrushchenko, N. V., Iova, A. S. (2014). Acoustic stress in premature neonates: causes of appearance and methods of correction. Bulletin of the North-Western State Medical University named after I.I. Mechnikov, 6 (2), 101-109.

[16] Gélat, P., David, A. L., Haqhenas, S. R., Henriques, J., Thibaut de Maisieres, A., White, T., Jauniaux, E. (2019). Evaluation of fetal exposure to external loud noise using a sheep model: quantification of in utero acoustic transmission across the human audio range. American Journal of Obstetrics and Gynecology, 221 (4), 343. doi: http://doi.org/10.1016/j.ajog.2019.05.036

[17] Sood, A. K., Singh, S. (2011). Vibroacoustic stimulation and modified fetal biophysical profile for early intrapartum fetal assessment. The Journal of Obstetrics and Gynecology of India, 61 (3), 291-295. doi: http://doi.org/10.1007/s13224-011-0044-5

[18] Safonova, I. N. (2016). Antenatal ultrasound monitoring and perinatal results in antepartum fetal distress. Eksperimentalnaya i klinicheskaya medicina, 1 (70), 131-138.

[19] Antonets, V. A., Kazakov, V. V. (2014). On noninvasive assessment of acoustic fields acting on the fetus. Acoustic magazine, 60 (3), 320-326. doi: http://doi.org/10.7868/s0320791914030022

[20] Đorđević, V., Panić, M., Stanojević, N. (2019). Risk Factors at Birth and their Impact on Prelingual and Early Lingual Development in Prematurus. 7-th International Conference on Fundamental and Applied Aspects of Speech and Language, 377-384. Available at: http://iefpg.org.rs/Conference/2019/S \%26L2019_Program_and_Abstracts.pdf 